\title{
NEW CONCEPT OF NUMERICAL SHIP MOTION MODELLING FOR TOTAL SHIP OPERABILITY ANALYSIS BY INTEGRATING SHIP AND ENVIRONMENT UNDER ONE OVERALL SYSTEM
}

\author{
Henryk Olszewski \\ The State University of Applied Sciences (PWSZ), Elbląg, Poland \\ H. Ghaemi \\ Gdańsk University of Technology, Poland
}

\begin{abstract}
The paper presents a new concept of overall ship motion modelling for application to total ship operability. The delivered model is a multi-phase and includes both submerged part of ship's hull and the surrounding water as a unique body. The Discrete Finite Element Method is applied. The model is successfully examined and illustrated for a selected AHTS
\end{abstract}

\section{INTRODUCTION}

The present analytical and numerical models of ship motions have been made so far based on a unique concept of dividing ship and environment into individual parts, modelling each of them separately and then analysing the ship motion under external forces and moments induced by wind, waves, currents or other steady or floating objects near to the ship by including hydrodynamic forces and moments generated due to ship velocity and acceleration [1-7].

In this paper, it is considered that there is only one total system which includes both the environment and ship, i.e. there is one multi-phase body with different rigid and elastic parts and its motion is modelled as a single body. The concept is simple but differs in philosophy of modelling in comparison to the present models. The model is numerical one worked out on the basis of the discrete finite elements method. The meshing is dynamic, changed at each step of simulation after linearization and varies from the dynamic meshing of today available software since the latter considers motion of boundary of the hull as a separate body. By combining the hull and environment under one total system it is possible to determine the added masses directly and identify interactions between solid and elastic elements of the system with no additional boundary conditions imposed upon the system. In practice, it means increasing the number of elements of the generated mesh and need of powerful computing facilities, but it generalizes and simplifies the model. 
The paper illustrates a part of results of a project related to the total ship operability. The presented model and results will be used for total ship operability analysis in further steps of the project.

Ships are examples of complex mechanical systems which perform specific tasks under coupled static and dynamic loads in the form of forces and moments generated by the ship motion or enforced externally. To assess the ship total operability it is necessary to generate a total model which includes all ship's subsystems, connected elements and her environment. Today, it is a usual practice to divide such a complex floating system into subsystems and then analyse each of them individually to assess the operability of the ship and its components [14]. An example of overall ship model is presented in Fig. 1.
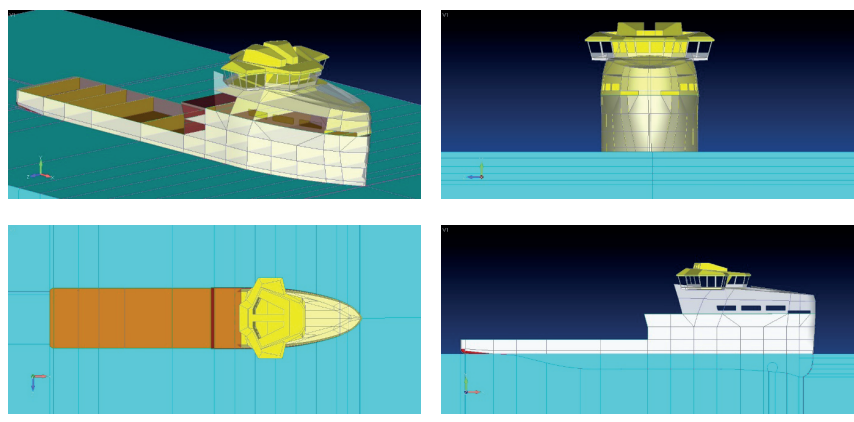

Fig. 1. Overall model of a ship at sea

The main subsystems of a ship motion model include ship's hull and its components, deck and auxiliary equipment, cargo, propulsion plant, control and navigation equipment, as well as model of waves, currents, wind and all other related environmental impacts. These subsystems may also include other complex mechanical, hydraulic, pneumatic, electrical or thermal complex elements which interact with the ship's environment. To analyse ship motion it is then necessary to work out a mathematical model which include all the subsystems and their elements. The first step of the modelling is therefore preparing a common mathematical model of ship hull and its environment. We focus now on such model in a numerical sense.

There are different methods of numerical modelling of the considered system. Solid Finite Elements Method (SFEM) [9], Deformation Finite Elements Method (DFEM) [10], Hybrid Finite Elements Method (HFEM) (a combination of SFEM and DFEM), Boundary Elements Method (BEM) and Discrete Finite Elements Method (DEM) are some examples which can be found in the literature. In practice, due to the complex dynamics of the ship's operability the interaction of individual subsystems is defined by the impact factors or coefficients $[13,14]$. Mutual interaction of partial subsystems can be initiated due to their different natural frequencies or those excited by enforced external inputs. Therefore, impact factors or coefficients should indicate the dependencies between the motion parameters of partial subsystems or forces which cause the motion. Generally, the impact factors depend on the motion of the subsystems.
For description of partial subsystems both linear and nonlinear models are used. In case of mutual interaction of partial subsystems described by linear models, impact factors are functions of forced vibration frequencies. The frequencies coincide with forcing frequencies. The impact factors depend then on natural vibration frequencies and damping. The subsystems described by non-linear models are analyzed in time domain. Mutual interaction of the subsystems can be determined by means of impulse functions. However, to use the impact factors for description of mutual interaction of non-linear models, linearization of the models is required [14].

Development of numerical methods as well as computational capacity of computers made it possible to work out a method for total modelling the ship together with its surrounding sea environment without necessity of dividing the model into partial subsystems. Fig. 2 presents the geometrical model of water mass, in which an outline of ship underwater part can be observed.

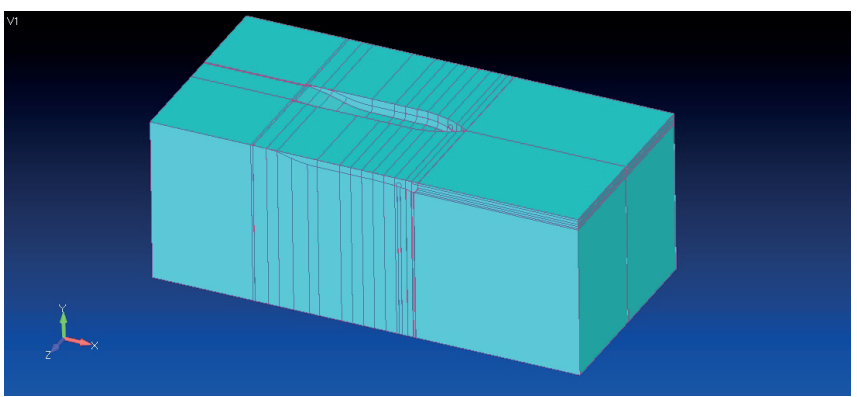

Fig. 2. Geometrical model of water mass - a part of total ship model

\section{MODELLING}

\section{GEOMETRY}

In case of the total modelling of ship operability a floating unit model may be defined at maintaining a required accuracy of mapping the ship's structure, as a result it is possible to analyze mutual interaction of between elements of hull structure, shipboard equipment and the environment. Fig. 3 shows a hull geometrical modelwith of a AHTS ship in this case.
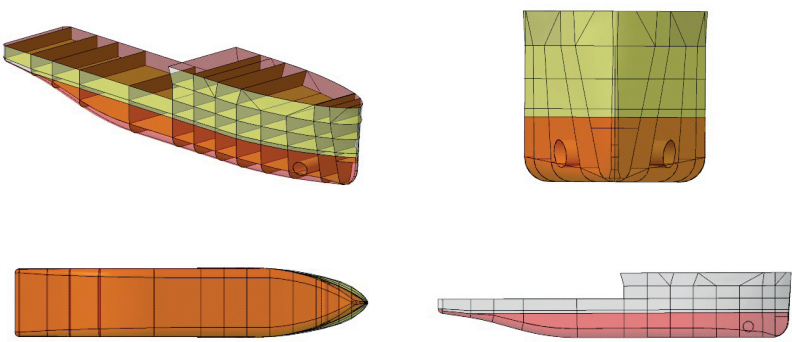

Fig. 3. Ship hull geometrical model 
The geometrical model of superstructure of the analyzed AHTS ship is shown in Fig. 4. The geometrical models assumed in this work constitute simplified physical systems representing real construction in the range of exactly determined mutual interactions. The simplifications were so assumed as to obtain deformations of ship and its equipment which differ from real ones in the range of a permissible error.
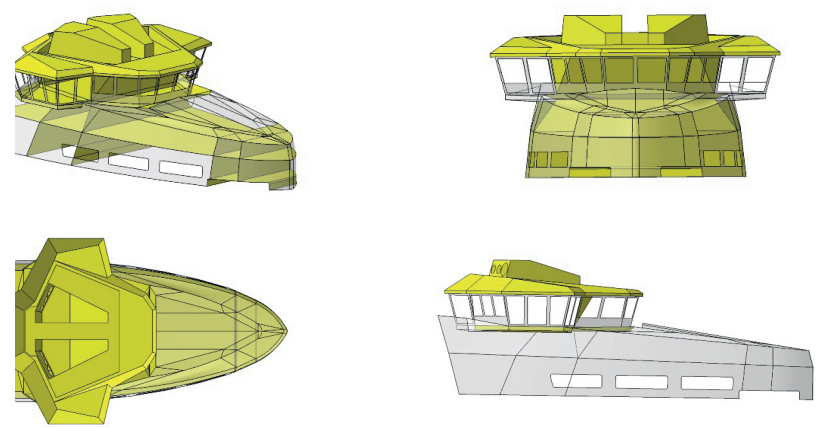

Fig. 4. Geometrical model of ship's superstructure

The ship together with its surrounding sea environment was subjected to digitization at maintaining full continuity of finite elements' mesh Fig. 5 and 6.

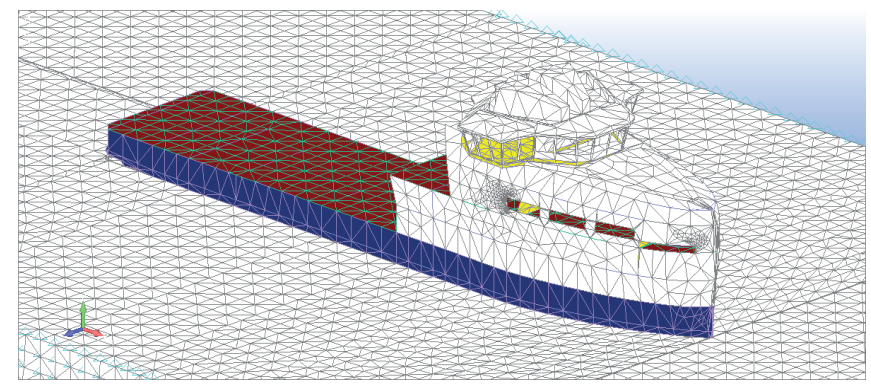

Fig. 5. Finite elements 'mesh of total ship model

\section{MASS DISCRETE MODEL}

For digitization of water mass the tetragonal elements (3D tetrahedron ones) were used. The tetragonal elements are simplexes in 3D Euclidean space, owing to this they make it possible to digitize arbitrarily complex geometrical spatial models, Fig. 6. To digitization of the complex geometrical model of the ship the triangular plate elements - simplexes in 2D Euclidean space were applied, Fig. 7.

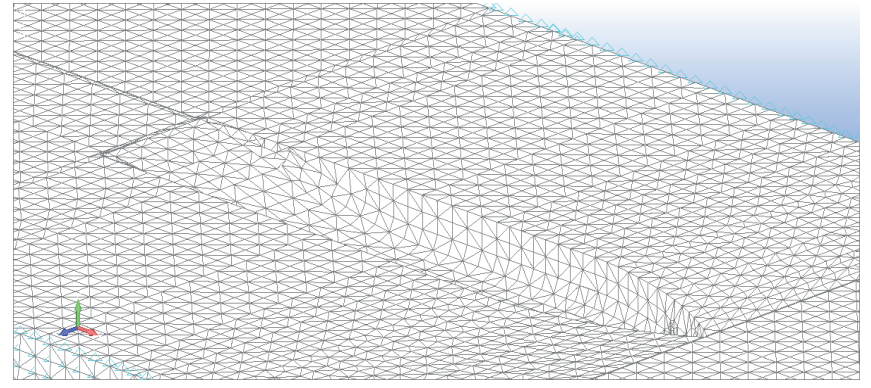

Fig. 6. Mesh of tetragonal finite elements of water with ship's hull "imprinted"in it.
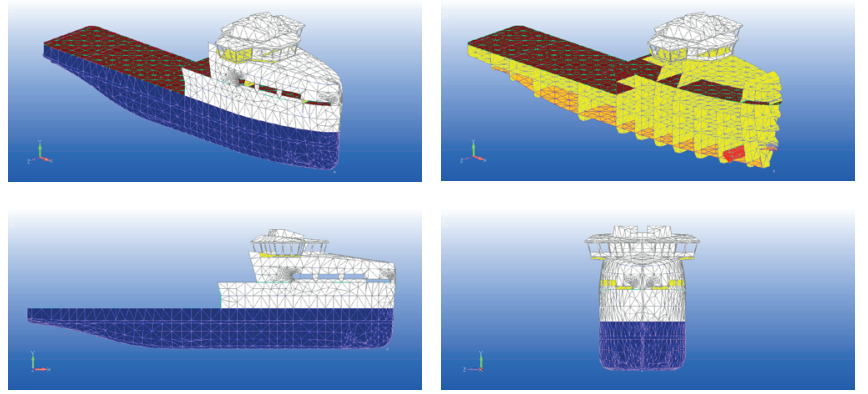

Fig. 7. Discrete model of the ship

In total modelling attention should be paid especially to making correct assumption of boundary conditions, sea environment parameters as well as parameters of ship machines and equipment.

In total modelling water waving and additional water mass result from analyzes but not assumptions as it is the case of classical modelling methods, Fig. 8.
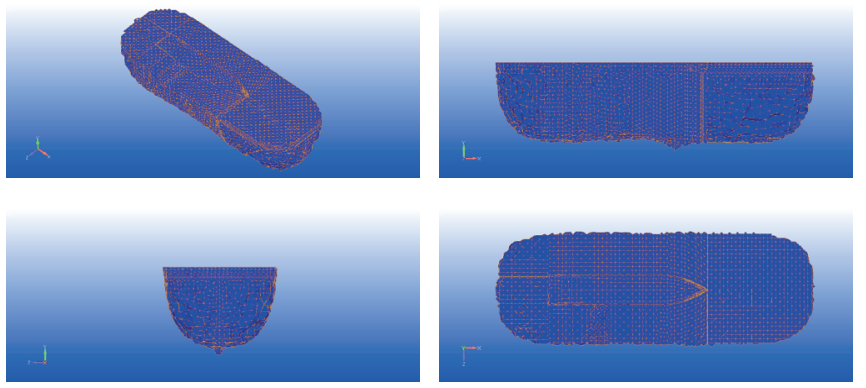

Fig. 8. Mesh of tetragonal finite elements of additional water mass

\section{ADDED MASS}

Digitization of water mass around floating unit made it possible to carry out 3D numerical calculations of water waving and analyzes of additional water mass in case when ship moves parallel to boundary of the analyzed area, Fig. 9. In the total model water mass is in direct contact with shell elements of underwater part of ship's hull therefore it is not necessary to assess assumed hydrodynamic quantities by means of e.g. the 3D Rankine SWAN (Ship Wave ANalysis) 
method worked out by the Laboratory for Ship and Platforms Flows, MIT, [11, 12].

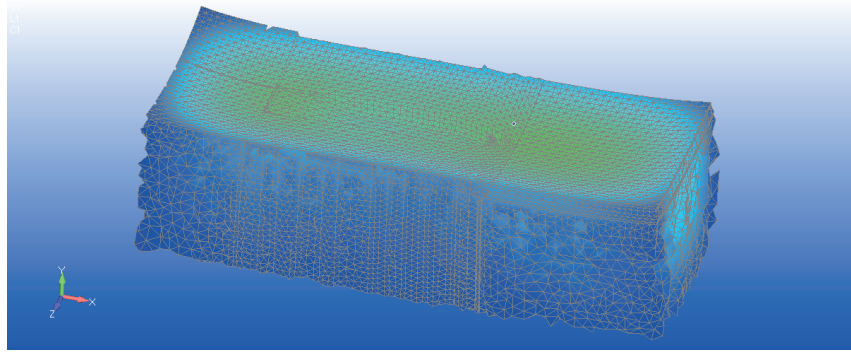

Fig. 9. Deformed mesh of finite elements of water mass around moving ship

Fig. 10 presents subsequent cross-sections of finite elements' mesh of additional water mass. Ship's movement excites displacements of fluid mass, greater in close neighbourhood of ship's hull. As the distance from the hull is growing the displacements of water are smaller and smaller, while the drop of the displacements is non- linear. It depends on distribution of physical properties of water mass as well as form of ship's hull - hence a geometrical non-linearity occurs.
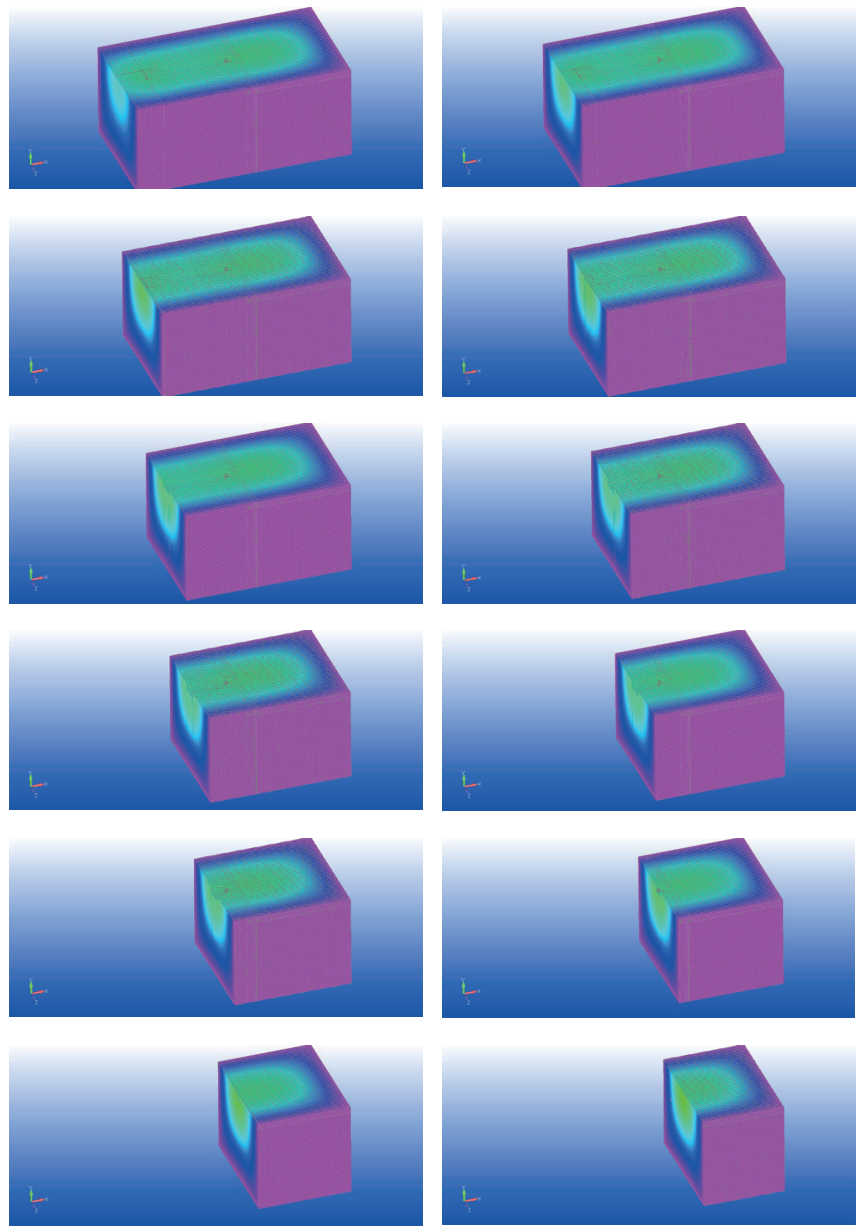

Fig. 10a. Cross-sections of additional water mass ( $1^{\text {st }}$ part)
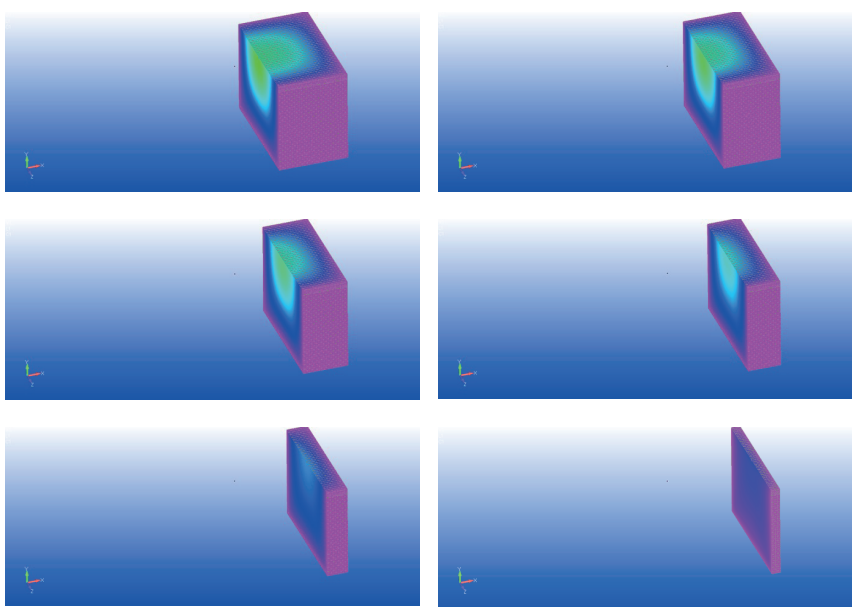

Fig. 10b. Cross-sections of additional water mass ( $2^{\text {nd }}$ part )

\section{DYNAMIC MESHING ADAPTATION}

For modelling and discretising of the vessel body, mainly the plate elements are used, see Fig. 7. An adaptive method for generating finite elements' mesh has been applied in order to make it denser in those regions, where sudden changes in ship hull form occur. In this way the finite elements' mesh is adjusted not only for the geometry of the model but also to generate the expected deformations (as the mesh was made denser in places where the largest deformations were expected). During discretisation of geometrical model special attention has been paid to proportions of dimensions of spatial elements; and as a result their height is comparable to their breadth and length, like in the case of plate elements, where their breadth is comparable to their length.

\section{MODEL ACCURACY}

On the one hand - and calculation duration time -on the other hand- are associated with the density of finite elements' mesh [10]. The required density of finite elements' mesh of the total model depends on many factors among which the following are crucial : shape of finite elements and digitized geometrical objects, expected increase of deformations, type of loads, boundary conditions, type of applied finite elements and their shape functions, and the required accuracy. In modelling and discretising of different objects, one should pay special attention to making the mesh denser in regions where sudden change in deformations is expected. The decreasing of dimensions of finite elements and increasing their number is called $h$-adaptive method used to increase calculation accuracy. Strain, displacement and stress energy allows to estimate accuracy of the performed calculations. Accuracy of calculations conducted by means of total modelling may be also determined by the error assessment method based on adopted standards [8]. Strain state inside a deformable finite element is determined on the basis of displacements of nodes which define the element (i.e. nodal quantities of the finite element ), constituting 
arguments of shape function. In the $h$-adaptive method the order of polynomial of finite element's shape function is not changeable. Such elements are called $h$-elements. Total model of floating unit's operability comprises: ship's model together with shipboard equipment, additional water mass model as well as air mass model (not considered in this paper). For modelling and discretising of the models different types of finite elements were used. Analysis of calculation results were based on nodal quantities of finite elements' mesh. In future analyzes the increasing of calculation accuracy is planned by rising order of shape function polynomials at maintaining shape of the mesh and dimensions of used finite elements. Such method is called $p$-adaptive one, and the elements used in it - p-elements.

The problem of assuming correct density of the mesh in total model was not the only which appeared during digitization of the geometrical model. Mesh discontinuity occurred in connections between finite elements of different types : shell elements with solid ones, triangular elements with quadrangular ones. The above mentioned transitions of finite elements' meshes were forced by geometry of modelled ship structure or necessity of joining solid elements digitizing water mass with plate and shell elements modelling underwater part of ship's hull. On account of the complex form of ship , its decks, bulkheads and equipment it was necessary to introduce intermediate stripes of finite elements to assure mesh continuity. The intermediate stripes of elements should not be applied to model regions to be analyzed in detail as well as to such parts of the mesh in which sudden changes in stresses and deformations are expected. The intermediate stripes of triangular elements ( triangular shell elements and tetragonal ones) were also used for modelling transitions from regions of rough mesh of finite elements to regions of dense mesh.

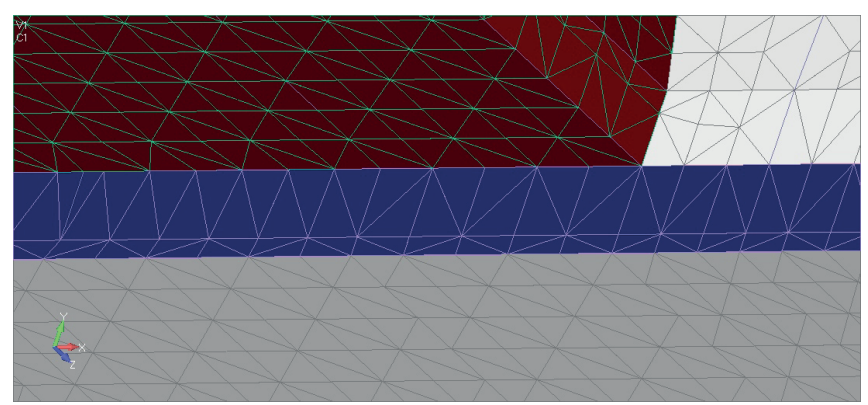

Fig. 11. Connection between plate elements of ship hull and tetragonal elements of water mass

In the course of modelling of underwater part of ship's hull a problem of making connection between plate elements of the hull and tetragonal spatial elements of water mass, appeared. The problem was associated with incompatible description of nodal quantities of the finite elements in question. The spatial elements have stiffness defined only for translational degrees of freedom while the plate elements - also for rotational degrees of freedom. The incompatibility of stiffness matrix of tetragonal elements and that of plate elements may lead to occurrence of solution singularity. There are many methods of joining together incompatible finite elements : it is possible to block surplus degrees of freedom or add intermediate elements to a discrete model : i.e. $R$-elements which transform rotations of a given node into displacements of neighbouring node.

\section{CONCLUSION}

The paper presents a new concept of overall ship motion modelling for application to total ship operability. The model is multi-phase one and includes both submerged part of ship's hull and the surrounding water as a unique body. The considered body includes both rigid and elastic elements with different parameters. It is assumed that the whole body moves with different speeds of its different elements. The DFEM is applied to make the numerical model of body motion. This modelling concept was successfully examined and illustrated for a selected AHTS. The main issues and challenges in making such model are discussed and the possible solutions are presented. In the next step of investigations the concept will be used to build multi-phase model of ship motion including air, additionally. Then natural frequency vibrations and forced motion will be analysed to pave the way for total operability analysis.

\section{REFERENCES}

1. Fossen, T. I.: Guidance and Control of Ocean Vehicles, John Wiley, 1994.

2. Fossen, T.I.: Marine Control Systems. Marine Cybernetics, 2002.

3. Perez, T.: Ship Motion Control. Springer Verlag, 2005.

4. Jensen, A. G.: Fluid dynamic derivatives: marine and wind engineering approaches. Journal of Wind Eng. and Industrial Aerodynamics 69(71),, 1997, pp. 777-793

5. Lewis, E.V.: Principles of Naval Architecture Vol. III: Motions in Waves and Controllability, $3^{\text {rd }}$ ed. Society of Naval Architecture and Marine Engineers, New York, 1998.

6. Price, W. C. and R. E. D. Bishop : Probabilistic theory of ship dynamics. Chapman and Hall, London, 1974.

7. Son, K.H. and K. Nomoto : On the coupled motion of steering and rolling of a high-speed container ship. Naval Architecture and Ocean Engineering, 1982.

8. Gawronski W.K.: Advanced Structural Dynamics and Active Control of Structures. Springer-Verlag New York Inc., 2004. 
9. Kruszewski J., Gawroński W., Wittbrodt E., Najbar F., Grabowski S.: Method of stiff finite elements (in Polish), Arkady, Warsaw 1975.

10. Larson M.G., Bengzon F.: The Finite Element Method: Theory, Implementation, and Practice. Springer-Verlag New York Inc., 2010.

11. Newman J.N.: Marine Hydrodynamics. The MIT Press, 1977.

12. Sclavounos P.D., Purvin S., Tahla U., Kim S.: Simulation based resistance and seakeeping performance of high-speed monohull and multihull vessels equipped with motion control lifting appendages. In Keynote Lecture, FAST 2003 Conference, Ischia Italy, 2003.

13. Walczyk Z., Kahsin M., Olszewski H.: Dynamic analysis of high power turbo set. 14th Internationale Tagung Forschung - Praxis - Didaktik im modernen Maschinenbau. Stralsund, 5.-8.5.2004. Stralsund: Fachhochsch. Stralsund (Univ. Appl. Sci.) Fachbereich Masch. 2004.

14. Walczyk Z., Olszewski H.: Dynamic properties of a structure supporting turbine set rotors (in Polish). Materials of $6^{\text {th }}$ School of modal analysis. Cracow, 11-12 December 2001, Ed. T.Uhl.: Department of Robotics and Machine Dynamics, AGH, Cracow 2001

\section{CONTACT WITH THE AUTHORS}

\author{
Henryk Olszewski \\ e-mail:h.olszewski1@wp.pl
}

The State University of Applied Sciences in Elbląg 1 Wojska Polskiego St.

$$
\text { 82-300 Elbląg }
$$

Poland

M. Hossein Ghaemi e-mail:ghaemi@pg.edu.pl

Gdańsk University of Technology 11/12 Narutowicza St. 80 - 233 Gdańsk

Poland 